\title{
Efficient Kinematic Solution to a Multi-robot with Serial and Parallel Mechanisms
}

\author{
Houxiang Zhang, Gionata Salvietti, Wei Wang, Guoyuan Li, Junzhi Yu, and Jianwei Zhang
}

\begin{abstract}
This paper presents an efficient kinematical solution to a multi-robot system with serial and parallel mechanisms. JL-I is a reconfigurable robot featuring active spherical joints formed by serial and parallel mechanisms endowing the robotic system with the ability of changing shapes in three dimensions. The active joint here can combine the advantages of the high rigidity of a parallel mechanism and the extended workspace of a serial mechanism. However, the kinematic analysis of the serial and parallel mechanism is always the bottleneck in designing a robot and control realization. In order to deal with this problem, the whole kinematical analysis is organized in the sequence from the direct mechanical analysis related to the serial and parallel mechanism over the numerical solutions to the simplified kinematics expression. The latest results obtained demonstrate that the deduced closure-form solution is time efficient and easy to implement while offering a satisfactory motion performance in on-site experiments.
\end{abstract}

\section{INTRODUCTION}

Mobile robots have the ability to move around in their environment and are not fixed to one physical location. Currently, mobile robots are the focus of a great deal of research and almost every major university has one or more labs that focus on mobile robotic topics. The modular approach makes the mobile robotic system versatile, robust, cost-effective and fast to prototype. The robots can adopt different configurations to match various tasks and suit complex environments [1], [2].

This paper presents the reconfigurable mobile robot JLI which currently comprises three identical modules (see Fig. 1). Actually, it is a general mobile platform and can be extended for various applications [3], [4]. Each single robotic unit in the JL-I system has reasonable locomotion ability. With the docking mechanisms, the units can connect to form a train-configuration which possesses a higher adaptability to rugged terrains. After docking, an equivalent spherical joints is relized via serial and parallel mechanism to enable the adjacent modules to adopt configurations by pitching, yawing, and rotating movements. However, the kinematic analysis of the serial and parallel mechanism is always

\footnotetext{
H. Zhang is with the Department of Informatics, University of Hamburg, Germany (Corresponding e-mail: hzhang@informatik.uni-hamburg.de).

G. Salvietti is a Ph.D candidate with the Department of Information Engineering, University of Siena, Italy.

W. Wang is with the Robotics Institute, Beijing University of Aeronautics and Astronautics, Beijing, China.

G. Li is a Ph.D candidate at TAMS, with the Department of Informatics, University of Hamburg, Germany.

$\mathrm{J}$. Yu is with the Institute of Automation, Chinese Academy of Sciences, Beijing, China.

J. Zhang is with the Department of Informatics, University of Hamburg, Germany.
}

the bottleneck in designing a robot and its control realization. This paper emphasizes the locomotion capabilities and reconfiguration realization. The remainder of the paper is organized as follows. In Section II, a brief overview of the application of the parallel and serial mechanism is presented. Section III is devoted to an efficient locomotion supported by a comprehensive workspace and kinematics analysis. Obtained experimental results are further illustrated in Section IV. Finally, Section V concludes the paper with an outline of future work.

\section{Applications of Serial and Parallel MECHANISMS IN ROBOTICS}

The serial mechanism has been adopted by mobile robots due to the extended workspace of the mechanism. Since the mechanical structure is always simple and direct, the movement control is easy to realize. In some cases, we call the traditional serial mechanism an open-loop mechanism. However, the advantages are offset by the low rigidity and the limited capability of payload. In order to realize a strong and powerful actuating joint only with serial mechanism, the whole mechanical design will be rather big and heavy.

With the development of mechanical technology, a parallel mechanism (PM) provides an alternative solution for mobile robots due to the higher positioning accuracy, higher control rigidity, and good dynamic behavior [5]. Note that the parallel mechanism is used firstly to realize robotic manipulators [6]. A generalized parallel manipulator is a closed-loop kine-

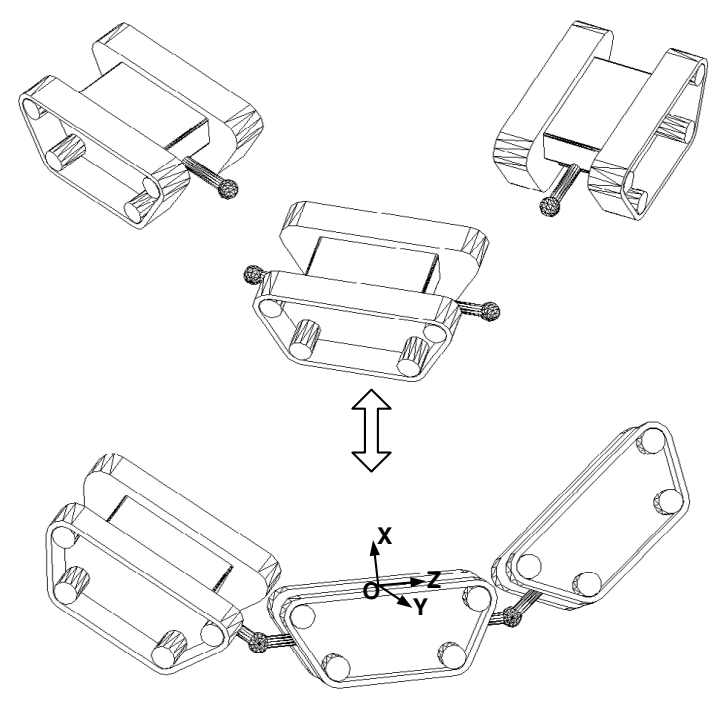

Fig. 1. The concept design of JL-I robot. 
matic chain mechanism whose end-effector is linked to the base by several independent kinematic chains. In many cases, the parallel mechanism owns several legs or limbs, each of them comprises of different kinds of joints in series, such as universal and prismatic joints. The Stewart mechanism as a well-known parallel design owns six limbs and six degrees of freedom [7]. As a result, it needs six actuators in total. The Stewart mechanism has been proposed for different robotic applications. Recently, many researchers have paid a lot of attention on researching the translational parallel manipulators and mechanisms due to only three degrees of freedom and outputs translational motion without changing its orientation [8]. Meanwhile, many alternative mechanical structures with less number of limbs have been proposed worldwide to overcome the shortcomings associated with sixlimb platforms, such as tripod [10].

There have been many research achievements in the design of manipulators [11] [12]. However, parallel mechanisms can only work in a very limited workspace even it is rather sophisticated. It is inevitable that the hybrid manipulator will be the possible idea that could integrate the advantages of both the parallel mechanism and serial mechanism. Some interesting prototypes are presented with the time. A hybrid (parallel serial) manipulator consisting of two serially connected parallel mechanisms is presented in [13], which gives its closed-form solution for the direct and inverse position problems. The other hybrid manipulator in [14] is with a base and two platforms in series.

Recently, there has been an increasing interest using parallel mechanism for designing mobile robots [15]. A reconfigurable parallel mobile robot which can be configured to $4 \mathrm{R}$ and $5 \mathrm{R}$ closed kinematical chains is proposed in [16]. The robot can form three-dimensional structures and reach a certain height. However these advantages are gained at the expense of a reduced workspace, difficult mechanical design and more complex kinematics and control algorithms [17]. As a result, researchers are beginning to combine the classical serial structure with parallel mechanism approach for the mobile robots' design too. The hybrid approach can overcome the limited workspace of the parallel mechanism and can provide features of both the serial and parallel mechanism. Currently, a lot of similar research can be found worldwide.

The JL-I robot presented in this paper owns three uniform units. One module is about $43 \mathrm{~cm}$ long, $25 \mathrm{~cm}$ wide and $15 \mathrm{~cm}$ high, and constitutes an entire robot system that can perform distributed activities [18]. The mechanical structure of the module comprises two powered tracks, a serial mechanism, a parallel mechanism and a docking mechanism. JLI features serial and parallel mechanisms to form a 3-DOF active spherical joint. By controlling the active joints and the docking mechanisms, the robot can change its shape in three dimensions. The serial mechanism can rotate $360^{\circ}$ around the $Z$ axis. The parallel mechanism can pitch around the $Y$ axis and yaw according to the $X$ axis.

Although some related work focusing on mechanical design, and control realization [19] on the JL robotic system has been presented previously, many important issues related to serial and parallel mechanism kinematics and inverse kinematics which particularly distinguishes the JL system from other similar robotic platforms have not been thoroughly investigated as yet. The following sections will elaborate on these issues.

\section{Analytical Solution to EfFicient LOCOMOTION}

\section{A. The DOF of the Active Joint}

It is noted that the calculations involved in performing a desired manipulation (forward kinematics) with a parallel mechanism are usually harder and have more than one unique solution. To achieve this reconfiguring capability, some related issues on the kinematics analysis of two connected modules will be briefly reviewed. Fig. 2 demonstrates the kinematics model of the joint between two modules. The required orientation for the reference frame $O^{\prime} X^{\prime} Y^{\prime} Z^{\prime}$ on the back module is implemented by a rotation of $\theta_{z}$, a pitching angle $\theta_{y}$ and a yawing angle $\theta_{x}$ according to the relative axes. Actually the pitching and yawing motions are implemented by the outstretching and returning movement of the $L_{1}, L_{2}$ of the parallel mechanism, and the rotation of $\theta_{z}$ is actuated by the serial mechanism. The freedom of the reconfiguring movement is three and can be represented by a generalized coordinate $\theta,(1)$. The joint variants of the movement are defined as $q$, (2).

$$
\begin{gathered}
\theta=\left[\theta_{x}, \theta_{y}, \theta_{z}\right]^{T} \\
q=\left[L_{1}, L_{2}, \theta_{z}\right]^{T}
\end{gathered}
$$

As can be observed from Fig. 2, after docking, the JL system has eight joints as a whole. Three Hooker joints are at points $O, A$, and $B$; two linear movement joints are at links $A C$ and $B D$; one rotating joint is along the axis $Z_{1} Z_{2}$ and two spherical joints are at $C$ and $D$. According to the mechanical principle, the DOF can be calculated as follows:

$$
m=6(n-g-1)+\sum_{i=1}^{g} f_{i}=3
$$

where $m$ stands for the DOF; $n$ denotes the movable links of the active joint; $g$ indicates the total number of the joints; and $f_{i}$ is the DOF of the relative joints. The details of the DOF analysis can be found in [20].

As mentioned above, the angle $\theta_{z}$ is required to have a $360^{\circ}$ rotation around the $Z$ axis. Note that $\theta_{z}$ is an independent DOF actuated by the serial mechanism and it normally occurs after the pitching movement or yawing movement in practice. So we only need to focus on the workspace of $\theta_{x}$ and $\theta_{y}$ since both pitching and yawing movements are dependent on the extending or contacting cooperation of $L_{1}$ and $L_{2}$ in the parallel mechanism.

In practice, the maximum positions should be calculated considering the mechanical constraints and collision avoidance. The pitching and yawing workspaces are obtained, 


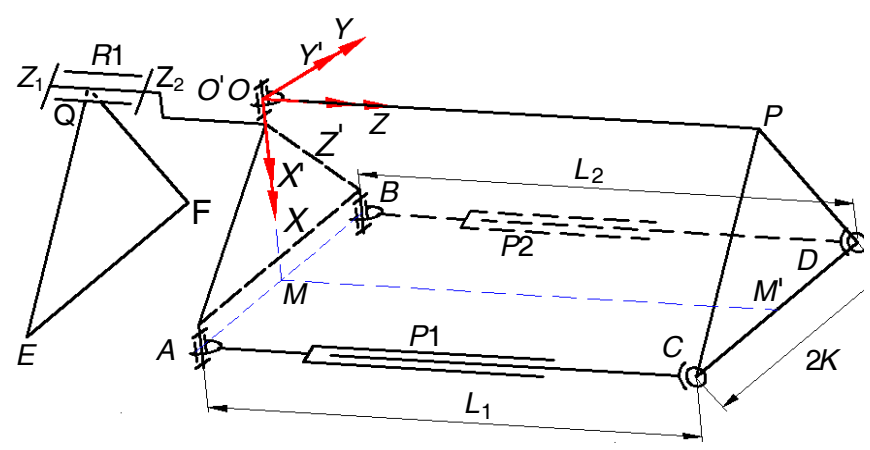

Fig. 2. The kinematics mode of the active spherical joint.

details can be found in [20]. The restricted workspace for avoiding collision is shown in (4).

$$
\left\{\begin{array}{l}
\theta_{x} \in\left[-32.0^{\circ},-8.0^{\circ}\right] \cup\left[+8.0^{\circ},+32.0^{\circ}\right] \\
\theta_{y} \in\left[-50.0^{\circ},-24.0^{\circ}\right] \cup\left[+24.0^{\circ},+50.0^{\circ}\right]
\end{array}\right.
$$

To further simplify the mechanical structure, we can design the workspace of $\theta_{x}, \theta_{y}$ to be the same, e.g., both within $-50-50^{\circ}$, which not only reduces the implementation cost but also slightly increases redundancy for practical operation.

\section{B. Direct and Inverse Kinematics Analysis}

The relationship between $q$ and $\theta$ was deduced in our previous paper [1] and is shown in (5) and (6), which will be useful for calculating the value of $q$ from the known generalized coordinate $\theta$ in motion control.

$$
\begin{aligned}
L_{1}^{2}= & {\left[K\left(\cos \theta_{y} \cos \theta_{z}+\sin \theta_{x} \sin \theta_{y} \sin \theta_{z}\right)\right.} \\
& -K\left(-\sin \theta_{z} \cos \theta_{y}+\sin \theta_{x} \sin \theta_{y} \cos \theta_{z}\right) \\
& \left.+L \cos \theta_{x} \sin \theta_{y}-K \cos \theta_{z}-K \sin \theta_{z}\right]^{2} \\
& +\left[K\left(\cos \theta_{x} \sin \theta_{z}\right)-K\left(\cos \theta_{x} \cos \theta_{z}\right)\right. \\
& \left.-L \sin \theta_{x}-K \sin \theta_{z}+K \cos \theta_{z}\right]^{2} \\
& +\left[K\left(-\sin \theta_{y} \cos \theta_{z}+\sin \theta_{x} \cos \theta_{y} \sin \theta_{z}\right)\right. \\
& -K\left(\sin \theta_{y} \sin \theta_{z}+\sin \theta_{x} \cos \theta_{y} \cos \theta_{z}\right) \\
& \left.+L \cos \theta_{x} \cos \theta_{y}\right]^{2} \\
L_{2}^{2}= & {\left[K\left(\cos \theta_{y} \cos \theta_{z}+\sin \theta_{x} \sin \theta_{y} \sin \theta_{z}\right)\right.} \\
& +K\left(-\sin \theta_{z} \cos \theta_{y}+\sin \theta_{x} \sin \theta_{y} \cos \theta_{z}\right) \\
& \left.+L \cos \theta_{x} \sin \theta_{y}-K \cos \theta_{z}+K \sin \theta_{z}\right]^{2} \\
& +\left[K\left(\cos \theta_{x} \sin \theta_{z}\right)+K\left(\cos \theta_{x} \cos \theta_{z}\right)\right. \\
& \left.-L \sin \theta_{x}-K \sin \theta_{z}-K \cos \theta_{z}\right]^{2} \\
& +\left[K\left(-\sin \theta_{y} \cos \theta_{z}+\sin \theta_{x} \cos \theta_{y} \sin \theta_{z}\right)\right. \\
& +K\left(\sin \theta_{y} \sin \theta_{z}+\sin \theta_{x} \cos \theta_{y} \cos \theta_{z}\right) \\
& \left.+L \cos \theta_{x} \cos \theta_{y}\right]^{2}
\end{aligned}
$$

However, the direct relationship or kinematics is still unclear. Normally a direct kinematics solution is hard to find because of multiple possible solutions due to the characteristics of extension movements of the parallel mechanism. A numerical method instead is used to resolve the problem so that the required workspaces of joint movements can be plotted in green as shown in Fig. 3.

The practical workspace of $L_{1}$ and $L_{2}$ should be within the surfaces marked $E_{1} F_{1} G_{1} H_{1}$ and $E_{2} F_{2} G_{2} H_{2}$, respectively,
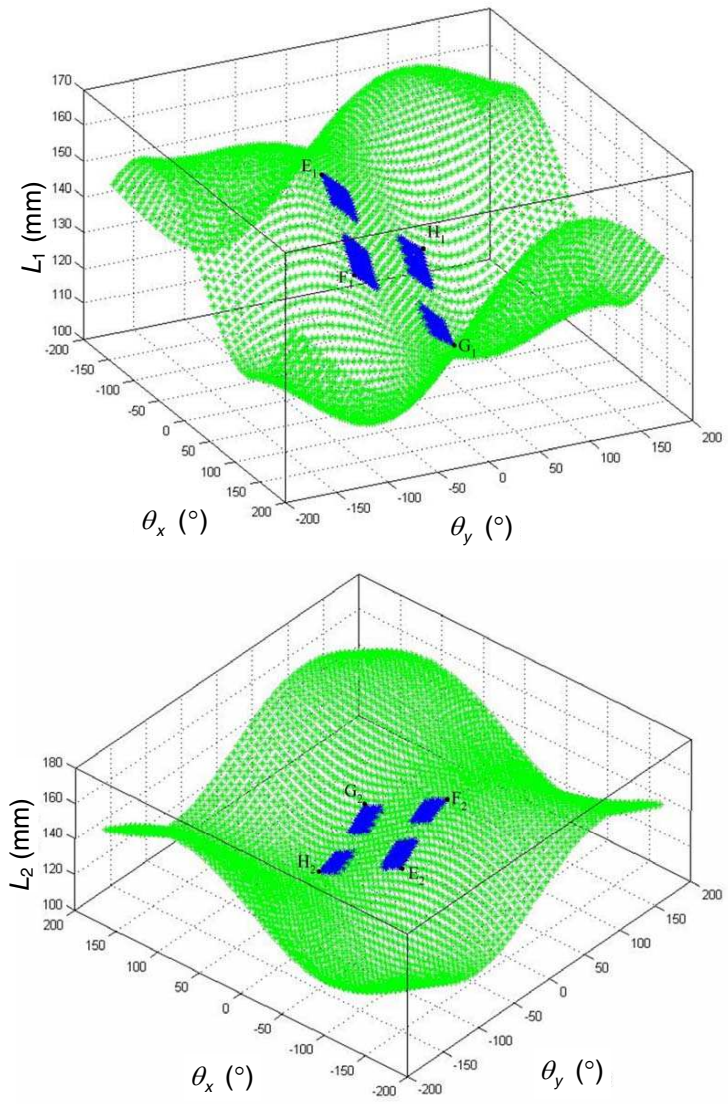

Fig. 3. The 3D plots of $L_{1}$ and $L_{2}$ according to $\theta_{x}$ and $\theta_{y}$ satisfying the constraints of (4). The green parts show the 3D plots of $L_{1}$ and $L_{2}$ according to $\theta_{x}$ and $\theta_{y}$ within $180^{\circ}$.

as illustrated in Fig. 3 too. Furthermore, to achieve $90-180^{\circ}$ recovery movements in a smooth and successful manner, $\theta_{x}$ and $\theta_{y}$ can only be actuated within the four disperse zones in blue, which well correlates with the conclusion from (4).

In order to get a direct kinematics expression, for implementing the serial and parallel mechanism, an equivalent form derived from (5) and (6) when $\theta_{z}$ is zero, is described as:

$$
\left\{\begin{aligned}
d_{1}=\left\|L_{1}\right\|^{2}-\left\|L_{2}\right\|^{2}= & 4 K \sin \theta_{x}\left(K \sin \theta_{x}-L\right) \\
d_{2}=\left\|L_{1}\right\|^{2}+\left\|L_{2}\right\|^{2}= & 8 K^{2}+2 L^{2}-4 K L \sin \theta_{y} \cos \theta_{x} \\
& -4 K^{2} \cos \theta_{y}-4 K^{2} \cos \theta_{x}
\end{aligned}\right.
$$

where $\|$.$\| indicates the Frobenius norm and d_{1}$ and $d_{2}$ represent respectively yaw and pitch movements. From the viewpoint of control, an expression of $\theta_{x}$ and $\theta_{y}$ in the functions of $L_{1}$ and $L_{2}$ has to be provided. However equation (7) does not have a form-closure solution. The Jacobian matrix $J$ of this system is (8) so that a numerical solution is yielded using the Levenberg-Marquardt algorithm. Therefore, the transformation between $q$ and $\theta$ is solved so far. 


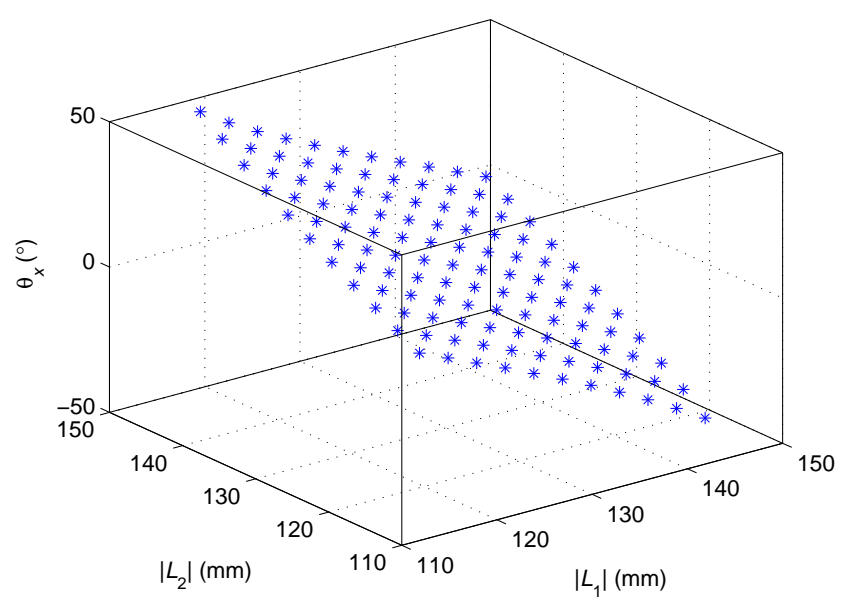

(a) $\theta_{x}$.

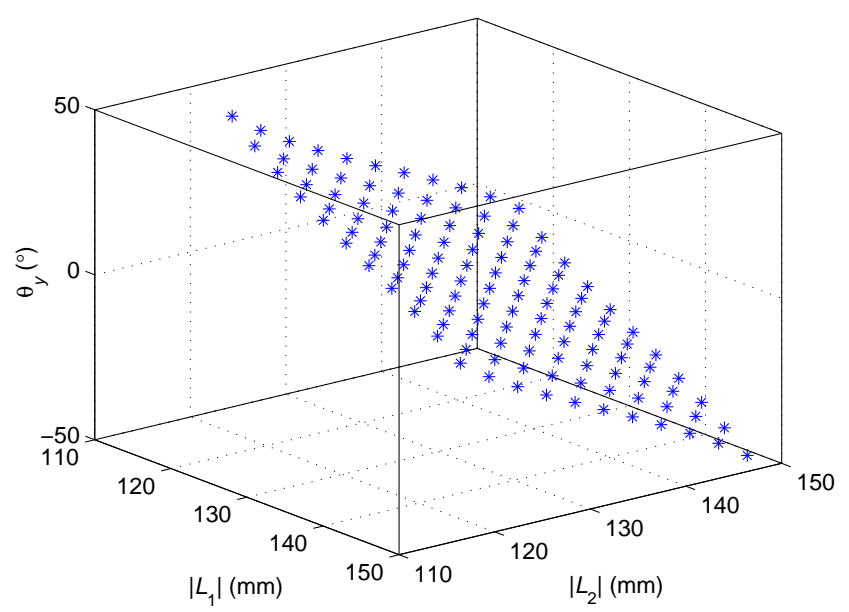

(b) $\theta_{y}$.

Fig. 4. The 3D plots of $L_{1}$ and $L_{2}$ according to $\theta_{x}$ and $\theta_{y}$ within $-50-$ $50^{\circ}$.

$$
\begin{gathered}
J=\left[\begin{array}{cc}
\frac{d\left(d_{1}\right)}{d \theta_{x}} & \frac{d\left(d_{1}\right)}{d \theta_{y}} \\
\frac{d\left(d_{2}\right)}{d \theta_{x}} & \frac{d\left(d_{2}\right)}{d \theta_{y}}
\end{array}\right] \\
=\left[\begin{array}{cc}
4 K c \theta_{x}\left(K s \theta_{y}-L\right) & 4 K^{2} s \theta_{x} c \theta_{y} \\
4 K L s \theta_{x} s \theta_{y}+4 K^{2} s \theta_{x} & -4 K L c \theta_{x} c \theta_{y}+4 K^{2} s \theta_{y}
\end{array}\right]
\end{gathered}
$$

A numerical method is often better to realize real-time kinematics. Fig. 4 provides an "actuation surface" which tells us all possible positions of $L_{1}$ and $L_{2}$ and the corresponding spatial angles $\theta_{x}$ and $\theta_{y}$.

For the convenience of control implementation, an expression of $L_{1}$ and $L_{2}$, and the corresponding spatial angles $\theta_{x}$ and $\theta_{y}$ has to be further provided. We remark that according to Fig. 4, it is complicated and time-consuming to calculate the outputs of the serial and parallel mechanism in order to actuate the module to an expected reconfiguration since a minimization problem is needed. In practice an approximation to a plane is possible to obtain a closure-form solution.

Since a planar relation is needed, it is possible to write it

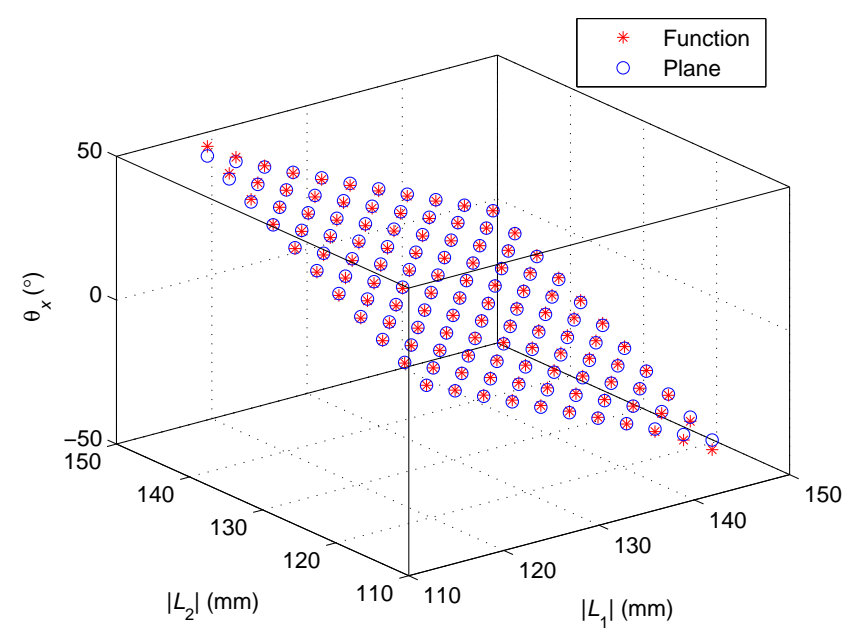

(a) $\theta_{x}$.

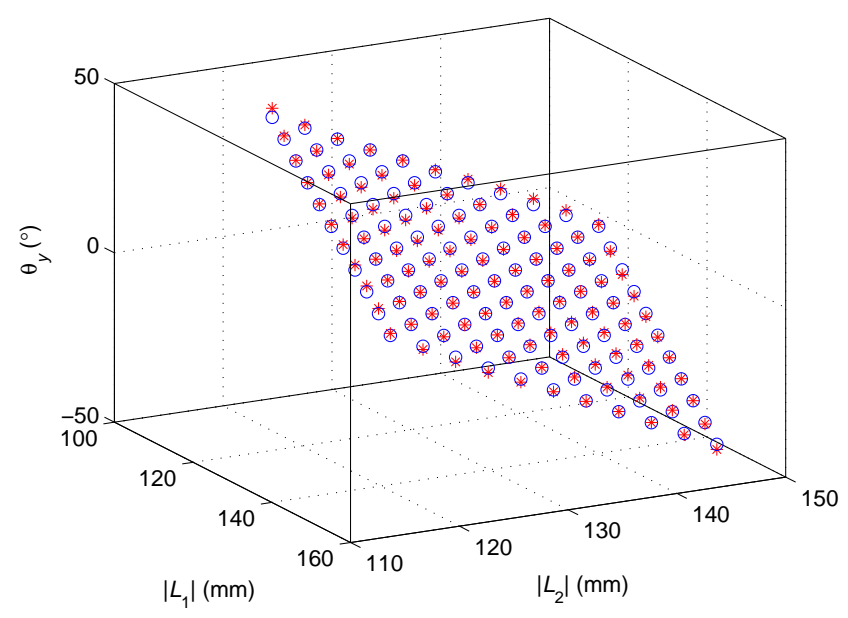

(b) $\theta_{y}$.

Fig. 5. The 3D plots of $\theta_{x}$ and $\theta_{y}$ within $-50-50^{\circ}$ based on (9).

as (9). The parameter vector $\left[\begin{array}{lllll}a & c & b & d & e\end{array}\right]^{T}$ can be computed using (10).

$$
\begin{gathered}
\left(\begin{array}{l}
\theta_{x} \\
\theta_{y}
\end{array}\right)=\left(\begin{array}{l}
a \\
d
\end{array}\right)+\left(\begin{array}{ll}
b & c \\
e & f
\end{array}\right)\left(\begin{array}{l}
L_{1} \\
L_{2}
\end{array}\right) \\
p=L_{\text {all }}^{+} \theta_{\text {all }}+N\left(L_{\text {all }}\right) \xi
\end{gathered}
$$

where $\theta_{\text {all }}=\left[\theta_{x} \theta_{y}\right]^{T}$ is the whole set of $\theta$ computed with the Levenberg-Marquardt algorithm; $L_{\text {all }}=\left[\begin{array}{ll}L_{x} & L_{y}\end{array}\right]^{T}$ is the whole set of the joint's lengths in the form $L_{x}=$ $\left[\begin{array}{llllll}1 & L_{1} & L_{2} & 0 & 0 & 0\end{array}\right]^{T}$ and $L_{y}=\left[\begin{array}{llllll}0 & 0 & 0 & 1 & L_{1} & L_{2}\end{array}\right]^{T} ; L_{\text {all }}^{+}$is the pseudo inverse of $L_{\text {all }} ; N($.$) represents the null space; \xi$ is a generic vector that lies in the null space of $L_{\text {all }}$. Fig. 5 shows the difference between the computed $\theta$ and the planar approximation. Once $p$ shown in (11) is computed out, $\theta$ can be expressed directly according to (9).

$$
p=\left[\begin{array}{llllll}
0.0 & -0.0264 & 0.0264 & 7.2198 & -0.0271 & -0.0271
\end{array}\right]^{T}
$$




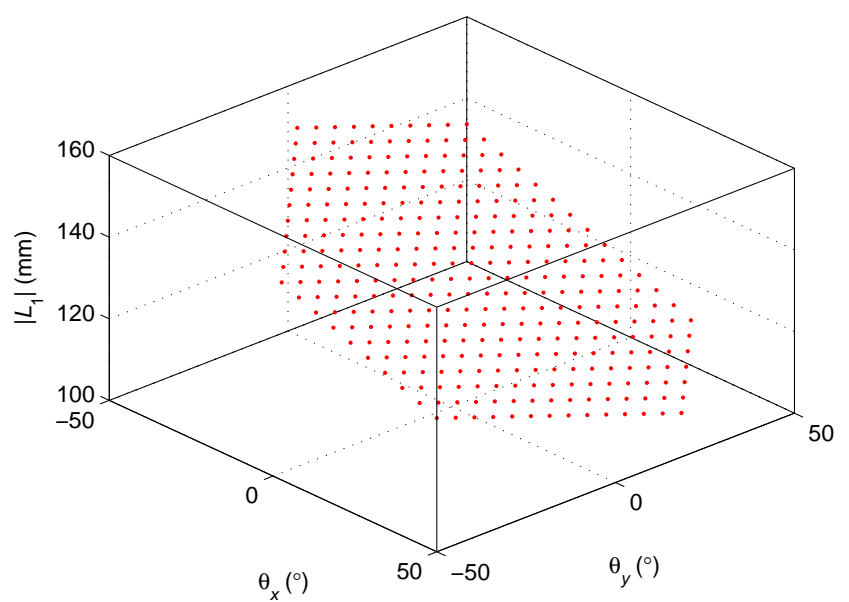

(a) $\left|L_{1}\right|$.

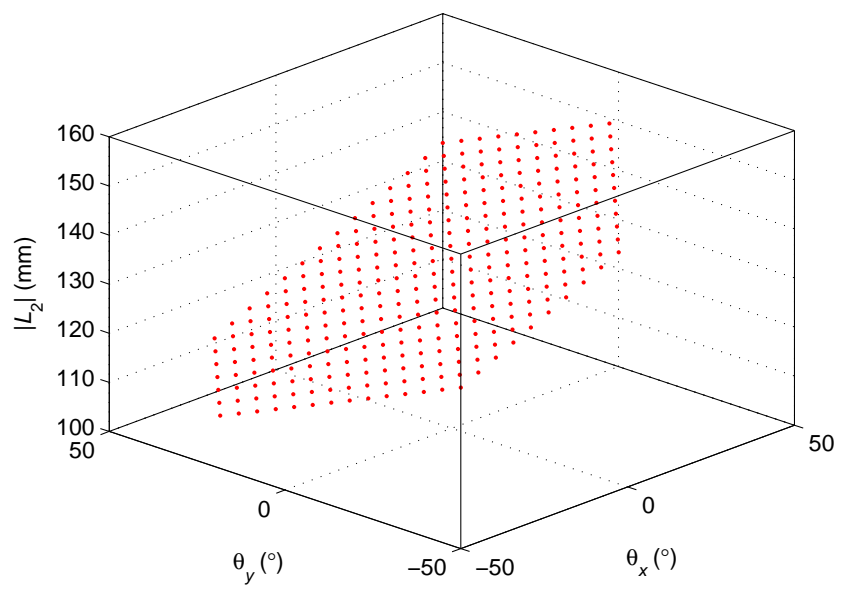

(b) $\left|L_{2}\right|$.

Fig. 6. The 3D plots of $L_{1}$ and $L_{2}$ according to $\theta_{x}$ and $\theta_{y}$ from (12).

In Fig. 5, the errors committed using the planar simplification are shown. The maximum error is around $3^{\circ}$ for $\theta_{x}$ and $\theta_{y}$, and the average errors are about $0.5^{\circ}$ for $\theta_{x}$ and $0.6^{\circ}$ for $\theta_{y}$. Evidently, the results are acceptable.

So far, the direct kinematics has been attained. Thus it offers an easy and quick solution for practical robotic design and control. In contrast, it is very simple to deduce an inverse kinematics expression (12) of the serial and parallel mechanism based on (9) when $p^{\prime}$ is $[-0.9777-$ $0.0036-0.1390-37.0358-0.1390-0.1354]^{T}$. The similar actuating plots of $L_{1}$ and $L_{2}$ according to $\theta_{x}$ and $\theta_{y}$ are easily calculated from (12), as shown in Fig. 6.

$$
\left(\begin{array}{l}
L_{1} \\
L_{2}
\end{array}\right)=\left(\begin{array}{l}
a^{\prime} \\
d^{\prime}
\end{array}\right)+\left(\begin{array}{ll}
b^{\prime} & c^{\prime} \\
e^{\prime} & f^{\prime}
\end{array}\right)\left(\begin{array}{c}
\theta_{x} \\
\theta_{y}
\end{array}\right)
$$

Up to now, a simplified efficient direct and inverse kinematics solution to the docking and posture-adjusting mechanical parts is given. The whole work is organized in the sequence from the direct mechanical analysis related to the serial and parallel mechanism over the numerical solutions to the simplified kinematics expression.

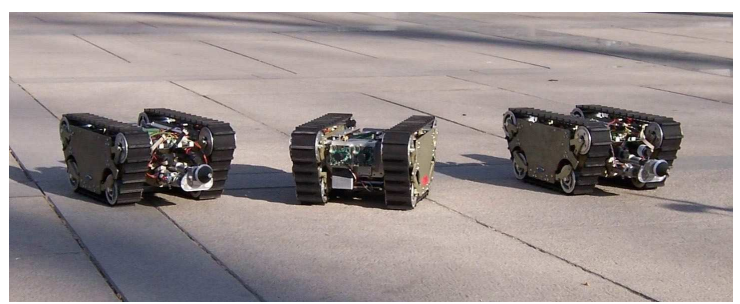

(a)

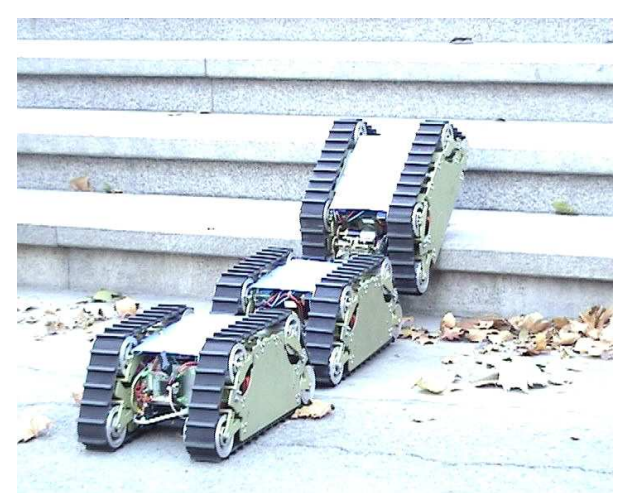

(b)

Fig. 7. Experimental scenarios of working alone (a) and collectively (b).

\section{EXPERIMENTS AND DISCUSSION}

\section{A. On-site Tests}

The research group has recently set up relevant robots and carried out successful on-site experiments with the mobile robot, confirming the principles described above and the robot's ability. Fig. 7 shows the system working alone and collectively in different environments. The working flexibility of the single robot is totally dependent on the mechanical design of the tracks and the motor's actuation, as shown in Fig. 7(a). While JL-I in the docked state is more robustly adaptable to rugged terrain than a single robot. The system can climb up high steps which are too difficult for one unit to deal with (Fig. 7(b)).

As mentioned previously, it is possible for JL-I to implement a recovering movement by adopting the proper configuration sequence on rugged terrain. Fig. 8 shows a series of actions of a $90^{\circ}$ self-recovery performed outdoors. Firstly JLI lay on the ground laterally. After a series of reconfigurable actions including pitching, yawing and rotating movements, JL-I could recover itself to a normal state again.

\section{B. Discussion}

During the experiment, the system was actuated successfully. On the one hand, from the control viewpoint the reconfiguration can be expected according to kinematics. Based on the amount of outputs of the joints, the locomotion implementation will be anticipated immediately in the world coordinate. More importantly, the simplified kinematics expression reduces the computation time and improves the working efficiency, thus making online runtime locomotion 


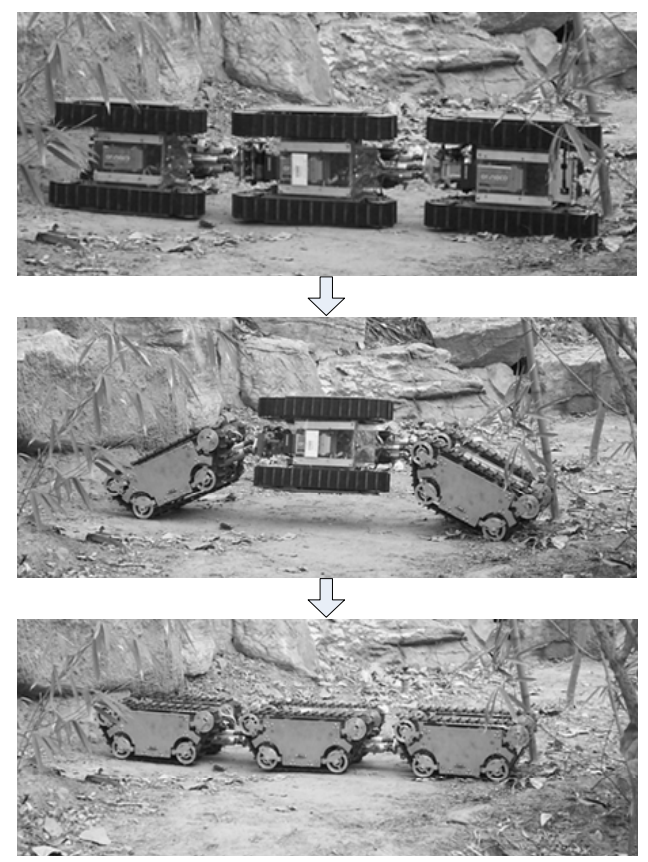

Fig. 8. Snapshots of the $90^{\circ}$ self-recovery.

and reconfiguration possible. As a result, the onboard control system of JL-I can be designed relatively simple and cheap.

According to the related analysis, the combination of parallel and serial mechanism could be designed compactly. First, the actuation working space of reconfiguration could be minimized, and then the mechanical structure of the parallel mechanism could be designed lightweight and smaller. Meanwhile, the efficiency of the online computing is maximized since all effective actuating solutions are in certain separated limited spaces. All related experiments confirmed the above analysis.

\section{CONCLusion}

This paper proposes a novel reconfigurable robot named JL-I featuring active spherical joints formed by serial and parallel mechanisms that endow the robot with the capability of changing shapes in three dimensions. The related kinematics issues are presented systematically. In practice, a direct kinematics expression and not only a numerical result is achieved. A simplified kinematics offers an easy and quick solution so that the movements can be assessed according to the joints' driving outputs. The analyzed results are essential for system design and even the implementation of the controlling mechanism for the robot.

\section{ACKNOWLEDGMENT}

The authors would like to thank the colleagues at the ARMS group at Beihang University (BUAA) for a great amount of work and on-site testing.

\section{REFERENCES}

[1] H. Zhang, Z. Deng, W. Wang, J. Zhang, and G. Zong, "Locomotion capabilities of a novel reconfigurable robot with 3 DOF active joints for rugged terrain," in Proc. IEEE/RSJ Int. Conf. Intell. Robot. Syst., Beijing, China, Oct. 2006, pp.5588-5593.

[2] M. Yim, D. Duff, and K. Roufas, "PolyBot: A module reconfigurable robot," in Proc. IEEE Int. Conf. Robot. Autom., San Francisco, USA, Apr. 2000, pp.514-520.

[3] F. Matsuno and S. Tadokoro, "Rescue robots and systems in Japan," in Proc. IEEE Conf. Robot. Biomim., Shenyang, China, Aug. 2004, pp.12-20.

[4] K. L. Paap, T. Christaller, F. Kirchner, "A robot snake to inspect broken buildings," in Proc. IEEE/RSJ Int. Conf. Intell. Robot. Syst., 2000, pp.2079-2082.

[5] R. Di Gregorio, "Kinematics of the translational 3-URC mechanism," J. Mech. Des., vol.126, no.6, pp.1113-1117, 2004.

[6] Y. Li, Q. Xu, "Design and development of a medical parallel robot for cardiopulmonary resuscitation," IEEE/ASME Trans. Mechatronics, vol. 12, no. 3, pp.265-273, 2007.

[7] K. Hunt, Kinematic geometry of mechanism. Clarendon Press, Oxford, 1978.

[8] M. Tanabe, Y. Takeda, "Kinematic Design of a Translational Parallel Manipulator with Fine Adjustment of Platform Orientation", Adv. in Mech. Eng., vol.2010, 2010.

[9] T. Huang, G. Tang, S. Li, Y. Li, G. Chetwynd, J. Whitehouse, "Kinematic calibration of a class of parallel kinematic machines (PKM) with fewer than six degrees of freedom," Sci. in China, vol.46, no.5, pp.515-526, 2003.

[10] R. Di Gregorio, "Statics and singularity loci of the 3-UPU Wrist," in Proc. Int. Conf. Adv. Intell. Mechatronics, Como, Italy, 8-12. July 2001, pp.470-475.

[11] M. Tsai, T. Shiau, Y. Tsai, T. Chang, "Direct kinematic analysis of a 3-PRS parallel mechanism", Mech. Mach. Theory, vol.38, pp.71-83, 2003.

[12] T. Itul, D. Pisla, "Workspace Analysis of a Three Degrees of Freedom Parallel Robot," in Proc. IEEE Int. Conf. Robot. Autom., Qual. Test. Rob., Cluj-Napoca, Romania, 25-28. May 2006, pp.290-295.

[13] T. Tanev, "Kinematics of a hybrid (parallel-serial) robot manipulator," Mech. Mach. Theory, vol.35, no.9, pp.1183-1196, 2000.

[14] L.Romdhane, "Design and analysis of a hybrid serialparallel manipulator," Mech. Mach. Theory, vol.34, no.7, pp.1037-1055, 1999.

[15] S. Moosavian, A. Pourreza, K. Alipour, "Dynamics and stability of a hybrid serial-parallel mobile robot", Math. Comput. Modell. Dyn. Syst., vol.3, no.1, pp.35-56, 2010.

[16] T. Yamawaki, T. Omata, O. Mori, " $4 \mathrm{R}$ and $5 \mathrm{R}$ parallel mechanism mobile robots", in Proc. IEEE Int. Conf. Robot. Autom., Barcelona, Spain, April 26-May 1, 2004, pp.3684-3689.

[17] X.Zheng, H.Bin, Y.Luo, "Kinematic analysis of a hybrid serial-parallel manipulator", Int. J. Adv. Manuf. Technol., vol.23, pp.925-930, 2004.

[18] H. Zhang, W. Wang, Z. Deng, and G. Zong, "A novel reconfigurable robot for urban search and rescue," Int. J. Adv. Robot. Syst., vol.3, no.4, pp.359-366, 2006.

[19] W. Wang, J. Qi, H. Zhang, G. Zong, "A rapid hunting algorithm for multi mobile robots system", in Proc. IEEE/RSJ Int. Conf. Ind. Electron. Appl., Harbin, Heilongjiang, China, May 2007, pp.12031207.

[20] H. Zhang, S. Chen, W. Wang, J. Zhang, and G. Zong, "Runtime reconfiguration of a modular mobile robot with serial and parallel mechanisms," in Proc. IEEE/RSJ Int. Conf. Intell. Robot. Syst., San Diego, USA, Oct. 2007, pp.2999-3004. 